\title{
Carcinoma verrugoso en paciente joven
}

\author{
Rosa LN*, Gedoz L**, Hildebrand LC, Carvalho A, Chevarría MG***
}

\section{RESUMEN}

El carcinoma verrucoso es una variación de bajo grado de malignidad del carcinoma de células escamosas oral, y la mayoría surge en la mucosa oral de individuos que mascan tabaco de forma crónica $(6,8,10)$. Estas lesiones encontradas predominantemente en hombres, con más de 55 años de edad (edad promedio de 65 a 70 anos) y las regiones de mayor frecuencia en mucosa bucal incluyen vestíbulo mandibular, mucosa yugal y palato duro $(6,8)$. La lesión aparece como una placa espesa, difusa, bien demarcada e indolora, con proyecciones superficiales papilares o verruciformes. Microscópicamente, se observa la presencia de crestas interpapilares anchas y alargadas y producción abundante de ceratina (2). La metástasis es un evento raro en los carcinomas verrucosos $(6,8,9)$. El tratamiento de elección es la excisión quirúrgica, asociada o no a la radioterapia. Los autores relatan un caso clínico de una paciente de 13 años de edad con lesión comprometiendo todo lo vermellion del labio inferior. El tratamiento inicial propuesto fue la radioterapia asociado a la quimioterapia, decido a la edad de la paciente, localización y extensión de la lesión. Después de una dosis total de 70Gy, hubo la regresión total de la lesión. Siendo así, el tratamiento del carcinoma verrucoso asociando a la radioterapia y quimioterapia se mostró adecuado en este caso.

Palabras clave: Carcinoma Verrucoso Oral, Radioterapia, paciente joven.

\begin{abstract}
The verrucous carcinoma is a low grade variant of oral squamous cell carcinoma, and it mostly appears in the oral mucosa of individuals who chew tobacco chronically $6,8,10$. The lesions are mainly found in men over 55 years old (average age 65 to 70 years) and the most common regions in the oral mucosa include the mandibular vestibule, jugal mucosa and hard palate 6,8 . The lesion appears as a well-defined painless diffuse thick plaque with superficial papillary or verruciform projections. Under the microscope the presence of wide elongated interpapillary ridges and copious keratin production are observed2. Metastasis is a rare event in verrucous carcinomas $6,8,9$. The treatment of choice is surgical excision, optionally combined with radiotherapy. The authors report a clinical case of a 13-year-old patient with a lesion compromising the whole lower lip vermilion. The initial treatment proposed was radiotherapy combined with chemotherapy, due to the patient's age and the localization and extent of the lesion. After a total dose of 70Gy, full regression of the lesion took place. Thus, treatment of verrucous carcinoma by combining radiotherapy with chemotherapy proved satisfactory in this case.
\end{abstract}

Key words: verrucous carcinoma, radiotherapy, child.

* Professor de Estomatologia da Facultad de Odontologia da Universidad Federal do Rio Grande do Sul.

** Professor Auxiliar de Patologia Oral da Facultad de Odontologia da Universidad Federal do Rio Grande do Sul.

*** Odontólogo alumno de Posgrado en Estomatologia da Facultad de Odontologia da Universidad Federal do Rio Grande do Sul.

Ambulatório de Estomatologia do Hospital de Clínicas de Porto Alegre.

Rosa LN, Gedoz L, Hildebrand LC, Carvalho A, Chevarría MG. Carcinoma verrugoso en paciente joven. Av. Odontoestomatol 2003; 19-3: 119-122. 


\section{INTRODUCCIÓN}

El carcinoma verrucoso es una variación de bajo grado de malignidad del carcinoma de células escamosas, presentándose como una lesión exofitica o como proyecciones papilares en la superficie de la mucosa (2). El primero a describirlo fue Ackerman en 1948, como una neoplasia asociada al hábito de mascar tabaco, pero ha sido diagnosticada en varios locales extraorales, tales como mucosa de la laringe, vagina, recto y piel $(1,2,6,7)$. A pesar de no ser una neoplasia muy infiltrativa, la lesión se extiende a los tejidos circunyacentes $(7,9)$. El tratamiento de elección para el carcinoma verrucoso es la excisión quirúrgica, sin la disección radical del cuello, una vez que la metástasis es extremadamente rara $(2,6)$. La radioterapia fue por mucho tiempo contraindicada, pues se corre el riesgo de desarrollo de carcinomas anaplásicos dentro de la lesión $(2,6,7,9,10)$.

El caso relatado a seguir impresiona por la rareza del tumor así como su aparecimiento en paciente joven sin estar involucrado con los factores etiológicos del carcinoma verrucoso. El tratamiento establecido para este caso fue la asociación de la radioterapia y quimioterapia debido a la extensión de la lesión, que en el momento del tratamiento ya comprometía todo el vermellion del labio inferior.

\section{CASO CLÍNICO}

En Junio de 1999 compareció para consulta en el Hospital de Clínicas de Porto Alegre (HCPA), la paciente CMT, género femenino, 13 años, leucoderma, natural y residente en la ciudad de Erechim, RS. La paciente se quejaba de dolores abdominales, cefalea, mareos y ictericia leve. La evaluaron, pero no obtuvo diagnóstico definitivo. En Junio de 2000, retornó al hospital con aumento de la ictericia y agravamiento de los dolores abdominales, nuevamente no se obtuvo diagnóstico definitivo. En la misma época la paciente relató el aparecimiento de lesión en el labio inferior semejante a una verruga y que el médico de su ciudad la había removido, pero sin examen histopatológico. Al final del mismo año a la paciente se la internó en la UTI - Pediátrica del HCPA debido al cuadro de hepatitis A autoinmune asociada a la leptospirosis. Después de un mes de internación (11/2000), aproximadamente 4 meses después de su remoción la lesión en el labio reapareció presentando dolor eventual y sangramientos frecuentes. Se diagnosticó clínicamente a la lesión como herpes simple y se la medicó con Aciclovir tópico y sistémico.

En Octubre de 2001 compareció nuevamente al HCPA para consulta en el Ambulatorio de Dermatología. La paciente seguía con la medicación tópica y la lesión del labio se presentaba ulcerada, con superficie costrosa, leve sintomatología, midiendo 1,5 x 1,5 cm de diámetro, localizada en el labio inferior en la región de la línea media. Presentaba también leucoplasia en el labio inferior alrededor de la lesión y en la mucosa yugal del lado derecho, además de adenomegalia cervical anterior derecha. Se suspendió la medicación tópica y se indicó la realización de biopsia incisional de la lesión del labio. El primer examen anátomopatológico no fue conclusivo, pues abarcó apenas área de necrosis, por eso se repitió el procedimiento cuyo resultado fue

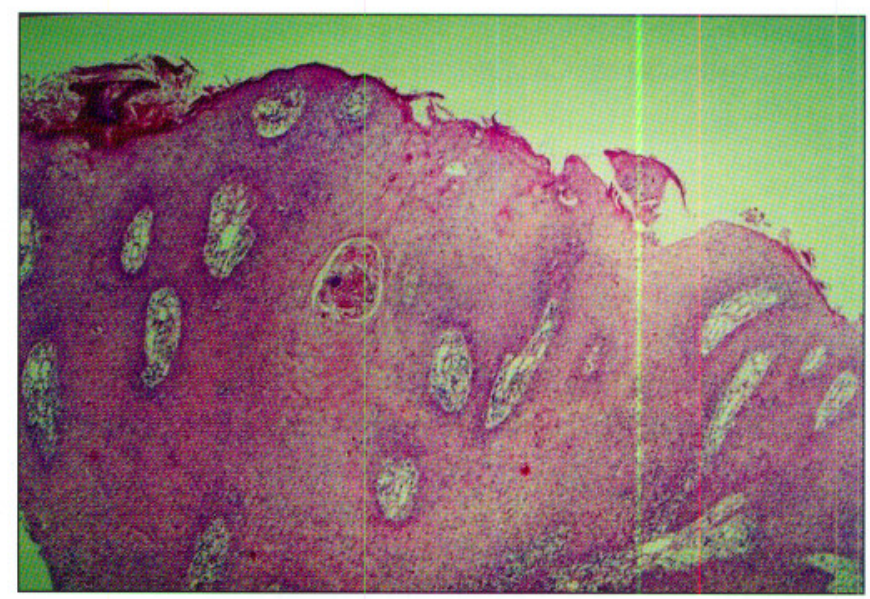

Fig. 1. Aspecto histopatológico.

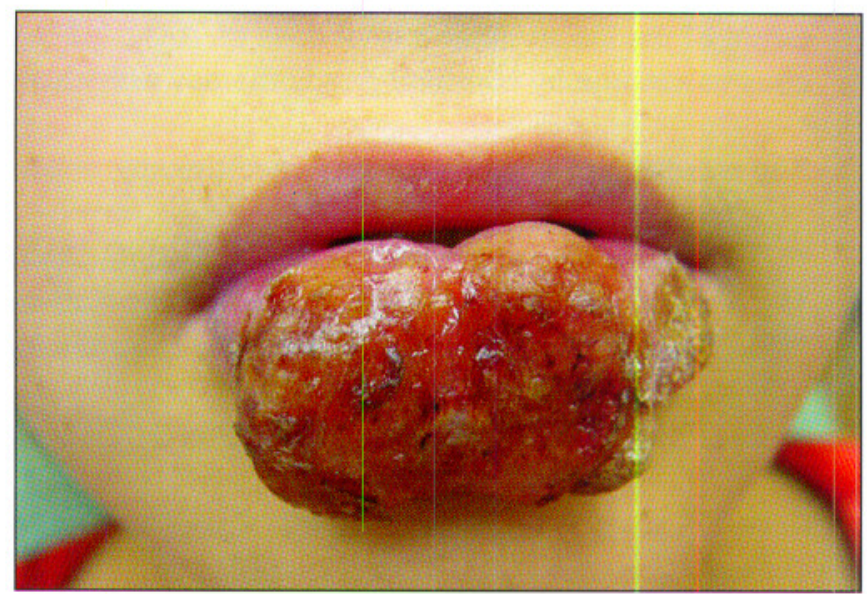

Fig. 2. Aspecto clínico inicial del paciente. 
sugestivo de carcinoma verrucoso. Para confirmar el diagnóstico se realizó otra biopsia, cuyo resultado del examen histopatológico fue de carcinoma verrucoso (Fig. 1). La paciente no presentaba metástasis, apenas nódulos inflamatorios en la cadena yúgulo - carotidea y región submandibular.

El tratamiento se lo inició en Enero de 2002 cuando a lesión ya comprometía todo el vermellion y parte de la mucosa del labio inferior, midiendo 4,0 x 2,0 cn (Fig. 2). El protocolo establecido para el caso fue Radioterapia asociada a la Quimioterapia. La radioterapia fue dividida en 35 sesiones, con dosis de 2 Gy en cada ciclo y dosis total de 70Gy (feixe AL e- $8 \mathrm{Mev}$.), incidiendo directa y únicamente sobre el tumor. Se inició el 21/01/2002 con término el 17/04/2002. La paciente desarrolló severa mucositis en el labio inferior (fig. 3) debido a la radioterapia, llevando a la suspensión del tratamiento dos veces. La quimioterapia realizada con

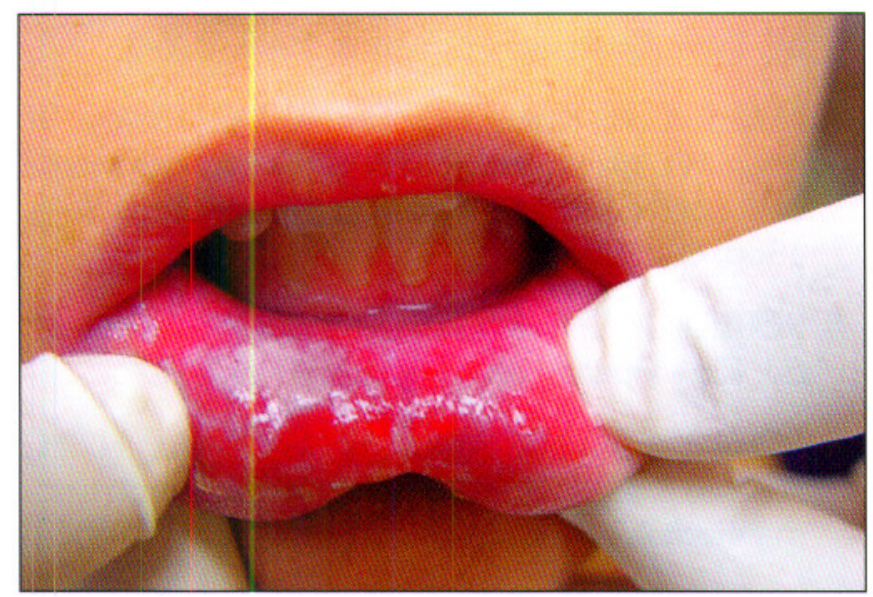

Fig. 3. Mucositis decorrente del tratamiento radioterápico.

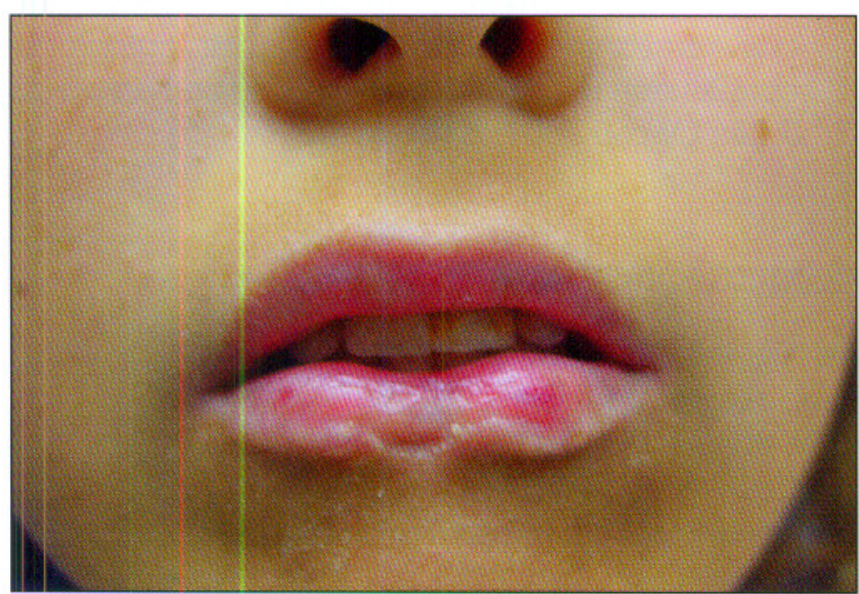

Fig. 4. Resultado final pos tratamiento. cisplatina (8 ciclos) tuvo como función apenas potencializar los efectos de la radioterapia. Durante y después del tratamiento a la paciente se la instruyó con higiene bucal, así como uso tópico de fluoreto de sodio $1 \%$ y visitas regulares al ambulatorio de Estomatología del HCPA para control de carie y lesiones bucales.

Después del tratamiento hubo remisión total del tumor (Fig. 4) estando la paciente en acompañamiento junto a la Oncología Pediátrica del HCPA.

\section{DISCUSIÓN}

El carcinoma verrucoso es una variación de bajo grado de malignidad del carcinoma de células escamosas (2). Lo describió por primera vez Ackerman en 1948, como una neoplasia asociada al hábito de mascar tabaco, pero ha sido diagnosticada en varios locales extraorales, tales como mucosa de la laringe, vagina, recto y piel $(2,6,7)$. En las lesiones intraorales se identificaron dos subtipos do papilomavirus humano (HPV), HPV-16 y HPV-18, pero su importancia todavía no está clara $(2,6,8,9)$.

La incidencia del carcinoma verrucoso representa 1 a $10 \%$ de los carcinomas de células escamosas orales, dependiendo del uso del tabaco en la población analizada. La mayoría de las lesiones surge en la mucosa oral de individuos que usan el tabaco de forma crónica. En individuos que poseen el hábito de mascar tabaco, normalmente la lesión surge en el local donde se coloca el tabaco $(2,6,9)$. Apenas $20 \%$ de las lesiones ocurren en pacientes no usuarios de tabaco en sus diferentes formas. Al carcinoma verrucoso se lo encuentra predominantemente en hombres mayores de la quinta década de vida. Su localización más frecuente es la región vestibular de la maxila, mucosa yugal, palato duro y blando, lengua y labio $(2,6,9)$. La lesión se presenta como una placa espesa, difusa, bien demarcada e indolora, con proyecciones superficiales papilares o verruciformes de crecimiento lento. Las lesiones son típicamente blancas, pero dependiendo de la cantidad de ceratina producida y de la reacción inflamatoria del hospedero al tumor pueden ser eritematosas o róseas. Se observa la presencia de áreas leucoplásicas en la superficie de la mucosa adyacente. A pesar de no ser una neoplasia muy infiltrativa, la lesión se extiende a los tejidos circunyacentes, y, cuan- 
do está localizado en la encía marginal se presenta fijada al periósteo $(6,7,9)$.

Microscópicamente el carcinoma verrucoso puede tener una apariencia engañadoramente benigna. se caracteriza por crestas interpapilares anchas y alargadas, que parecen empujar el tejido conjuntivo subyacente. Las lesiones normalmente presentan producción abundante de ceratina (paraceratina) y una superficie papilar o verruciforme. Las células epiteliales lesionadas generalmente muestran un patrón de maduración normal con ningún grado significativo de atipia celular. Se observa frecuentemente un intenso infiltrado de células inflamatorias crónicas en el tejido conjuntivo adyacente. El diagnóstico histopatológico del carcinoma verrucoso requiere una biopsia incisional adecuada, pues el patólogo necesita evaluar la configuración histopatológica general de la lesión (6).

El tratamiento de elección para el carcinoma verrucoso es la cirugía local, sin la necesidad del abordaje quirúrgico del cuello, una vez que la metástasis es extremadamente rara $(2,6)$. La radioterapia fue por mucho tiempo contraindicada, pues el carcinoma verrucoso no presenta una buena respuesta a este tipo de tratamiento y se corre el riesgo de desarrollo de carcinomas anaplásicos (2, 4, 6, 7, 9, 10). Según Koch et al. (2001), pacientes tratados quirúrgicamente tienen sobrevida mayor que aquellos tratados apenas con radioterapia, especialmente en las lesiones orales (5). Sin embargo estudios más recientes demostraron que el tratamiento radioterápico se ha mostrado efectivo en ciertos casos (9). Según levantamiento de Yoshimura et al. (2001), la cirugía es el método más confiable para el tratamiento del carcinoma verrucoso, sin embargo la radioterapia combinada con la quimioterapia es el tratamiento de elección cuando la primera es contraindicada (5). En estudio de Jyothirmayi et al. (1997), de los pacientes tratados apenas con radioterapia, $76 \%$ tuvieron remisión total de la lesión y en los casos de recidiva ninguno desarrolló carcinoma anaplásico (4). La quimioterapia utilizada aisladamente puede reducir temporalmente el tamaño de la lesión, pero no constituye un tratamiento definitivo (6).

\section{BIBLIOGRAFÍA}

1. ACKERMAN, L. V. Verrucous carcinoma of the oral cavity. Surgery. 1966; 23: 670-8.

2. CANNON, R; Hayne, S. T. Concurrent verrucous carcinomas of the lip and buccal mucosa. Southern Med J. 1993; 86 (6): 691-3.

3. ELLIOTT, G.B.; Macdougall, L.A.; Elliott, J.D.A. Problems of verrucose squamous carcinoma. Ann Surg. 1973; 177 (1): 21-9.

4. JYOTHIRMAYI, R.; Sankaranarayanan, R.; Varghese, C.; Jacob, R.; Nair, M. B. Radiotherapy in the treatment of verrucous carcinoma of the cavity. Oral Oncol. 2001; 33 (2): 124-8.

5. KOCH, B. B.; Trask, C. K.; Hoffman, H. T.; Karnell, L.H.; Robinson, R. A.; Zhen, W.; Menck, H. R. National survey of head and neck verrucous carcinoma: patterns of presentation, care and outcome. Cancer. 2001; 92 (1): 110-20.

6. NEVILLE, B. W.; Damm, D. D.; Allen, C. M.; Bouquot, J. E. Oral \& maxillofacial pathology. 1a ed. Rio - Guanabara Koogam. 1998; 296-8.

7. PEREZ, C. A.; Kraus, F. T.; Evans, J. C.; Powers, W. Anaplasic transformation in verrucous carcinoma of the oral cavity after radiation therapy. Am J Surg. 1966; 86: 108-15.

8. REGEZI, J. A.; Sciubba, J. J. Patologia Bucal. $1^{\text {a }}$. Ed. Rio - Guanabara koogan.1991: 124-7.

9. SCHWADE, J. G.; Wara, W. M.; Dedo, H. H.; Phillips, T. L. Radiotherapy for verrucous carcinoma. Radiology. 1976; 120: 677-9.

10. YOSHIMURA, Y.; Mishima, K.; Obara, S.; Nariai, Y.; Yoshimura, H.; Mikai, T. Treatment modalities for oral verrucous carcinomas and their outcomes: contribution of radiotherapy and chemotherapy. Int J Clin Oncol. 2001; 6 (4): 192-200.

\section{CORRESPONDENCIA}

Av. Nilópolis, 250/406

Porto Alegre - RS. Brasil

CEP: 90460-050

Teléfono: (51) 3316-8799

Correo electrónico: Inrosa@hcpa.ufrgs.br 